\title{
Infecciones osteoarticulares en un hospital pediátrico de alta complejidad: epidemiología y características clínicas asociadas con bacteriemia
}

\author{
Osteoarticular infections in a tertiary care children's hospital: \\ Epidemiology and clinical characteristics in association with \\ bacteremia
}

Dra. Esmeralda Highton ${ }^{a}$, Dra. M. Guadalupe Péreza ${ }^{a}$ Dra. Carola Cedillo Villamagua ${ }^{a}$, Dra. María I. Sormani ${ }^{a}$ Dra. María S. Mussinia, Bioq. Adela Isasmendib,

Técn. José Pinheiro ${ }^{b}$, Bioq. Vanesa Reijtman ${ }^{b}$, Dra. Moira Taicz ${ }^{a}$,

Técn. Alejandra Mastroianni ${ }^{b}$, Bioq. María E. García y Dra. María T. Rosanova ${ }^{a}$

\section{RESUMEN}

Introducción. Lasinfecciones osteoarticulares son una importante causa de morbilidad y pueden presentar bacteriemia. La epidemiología de estas infecciones se ha modificado en los últimos años. Objetivos. Describir las características epidemiológicas, clínicas y evolutivas de los niños con infecciones osteoarticulares y comparar los pacientes con bacteriemia con los que no la presentaron.

Población y métodos. Cohorte retrospectiva. Se incluyeron pacientes menores de 18 años, admitidos en el Hospital Juan P. Garrahan entre el 1/1/2016 y el 31/12/2016 con sospecha de infecciones osteoarticulares en quienes sehubiese realizado artrocentesis y/o biopsia articular. Se excluyeron niños con patología previa. Se compararon las características clínicas y de laboratorio según tuvieran bacteriemia o no. Se utilizó Stata 10.

a. Servicio de Control Epidemiológico e Infectología, Hospital de Pediatría "Prof. Dr. Juan P. Garrahan", Ciudad Autónoma de Buenos Aires, Argentina.

b. Servicio de

Microbiología, Hospital de Pediatría "Prof. Dr. Juan

P. Garrahan", Ciudad Autónoma de Buenos Aires, Argentina.

Correspondencia: Dra. M. Guadalupe Pérez: guaperez@hotmail.com

Financiamiento:

Ninguno que declarar.

Conflicto de intereses: Ninguno que declarar.

Recibido: 19-5-2017 Aceptado: 7-9-2017
Cómo citar: Highton E, Pérez MG, Cedillo Villamagua C, et al. Infecciones osteoarticulares en un hospital pediátrico de alta complejidad: epidemiología y características clínicas asociadas con bacteriemia. Arch Argent Pediatr 2018;116(2):e204-e209.

\section{INTRODUCCIÓN}

Las infecciones osteoarticulares (IOA) constituyen un desafío diagnóstico y de tratamiento para el pediatra. Son una causa frecuente de uso prolongado de antibióticos y de internación. Pueden provocar gran morbilidad, sobre todo, si el tratamiento inicial no es el adecuado. ${ }^{1}$ Son infecciones, en general, de manifestación aguda que pueden presentar bacteriemia en, aproximadamente, la mitad de los casos $^{2}$ y sepsis. ${ }^{3}$

Fuera del período neonatal, los principales agentes etiológicos son Staphylococcus aureus, Streptococcus pyogenes y Streptococcus pneumoniae. El aumento en la incidencia de Staphylococcus aureus meticilino resistente de la comunidad (SAMRco), el uso de técnicas moleculares de diagnóstico y la incorporación de vacunas al calendario oficial de vacunación han modificado la epidemiología de estas infecciones en las últimas décadas. ${ }^{4}$ La bacteriemia en las IOA ha sido descrita como marcador de gravedad. Se asocia con infecciones por Staphylococcus aureus meticilino resistente (SAMR) y sepsis. ${ }^{5}$ 
Es fundamental el diagnóstico precoz de las IOA y el inicio del tratamiento empírico adecuado para disminuir la morbilidad posterior. Se requiere el conocimiento actualizado de la epidemiología de este tipo de infecciones para adecuar el tratamiento empírico. Aun cuando el manejo es el correcto, un número significativo de casos puede resultar en un daño irreversible de la articulación.

El objetivo de este trabajo fue describir las características epidemiológicas, clínicas y evolutivas de los niños con IOA provenientes de la comunidad admitidos en un hospital pediátrico de tercer nivel de atención y comparar las características de los pacientes con bacteriemia con los que no la presentaron.

\section{POBLACIÓN Y MÉTODOS}

Se llevó a cabo un estudio de cohorte retrospectivo. Se incluyeron pacientes mayores de 1 mes y menores de 18 años, admitidos en un hospital pediátrico de alta complejidad desde el $1 / 1 / 2016$ al 31/12/2016 con sospecha de IOA en los que se realizó un procedimiento diagnóstico (biopsia ósea o sinovial y/o artrocentesis). Se excluyeron los niños con antecedente reciente de internación, patología osteoarticular previa y / o inmunocompromiso.

Se revisaron las historias clínicas de todos los pacientes que cumplieran con los criterios de inclusión. Se registraron antecedentes clínicos, parte quirúrgico y estudios de laboratorio realizados.

Se evaluaron la edad, días de evolución previos a la consulta, presentación clínica, diagnóstico presuntivo al ingresar, examen físico, localización/es de la lesión, tipo y número de drenajes quirúrgicos (por artrocentesis, artrotomía o artroscopia), días de evolución al momento del drenaje y días de internación.

Se registraron variables de laboratorio, cultivos del líquido articular y hemocultivos y la susceptibilidad antimicrobiana de los agentes involucrados.

Se documentó la necesidad o no de internación en la Unidad de Cuidados Intensivos, el tratamiento antimicrobiano empírico y definitivo y su duración tanto oral como endovenoso, la respuesta al tratamiento y la aparición de secuelas dentro de los 3 meses de seguimiento.

Las muestras de hemocultivos y líquido articular o biopsia ósea o sinovial se procesaron en el Laboratorio de Microbiología del Hospital.

Los hemocultivos fueron procesados mediante el sistema automatizado Bact/Alert 3D y, posteriormente, tipificados mediante pruebas microbiológicas convencionales y automatizadas, siguiendo los protocolos de trabajo vigentes en el Laboratorio de Microbiología.

Las muestras de líquido articular y biopsia se sembraron en agar sangre, agar chocolate, agar cistina-lactosa deficiente en electrolitos (CLDE) y caldo tioglicolato, y fueron incubadas a $37^{\circ} \mathrm{C}$ por un período de 24 a $72 \mathrm{~h}$. La identificación bacteriana se llevó a cabo mediante MALDITOF MS (Vitek-MS, Bio-merieux). Las pruebas de sensibilidad a los distintos antimicrobianos se realizaron por métodos automatizados (Vitek $2 \mathrm{C}$, Bio-merieux) y por el método de difusión con discos, según las normas del Clinical and Laboratory Standards Institute (CLSI). ${ }^{6}$

\section{Definiciones}

Tratamiento empírico: tratamiento antimicrobiano indicado antes de la identificación de un agente etiológico.

Tratamiento definitivo: antibiótico prescrito con los resultados de hemocultivos y / o líquido articular.

Infección adquirida en la comunidad: infecciones que se presentan en pacientes que no hayan estado internados en los últimos 6 meses ni concurran, al menos, semanalmente a algún centro de los cuidados de la salud.

Uso de antibióticos en la última semana: antecedente de haber recibido, al menos durante 48 h, algún antimicrobiano por vía sistémica (oral o parenteral).

Todos los estudios realizados fueron parte de la atención médica habitual para la patología y evolución de los pacientes. El análisis y publicación de los datos se realizó con el consentimiento y resguardando la identidad de los pacientes y sus familias. El protocolo fue presentado al Comité de Investigación y Ética del Hospital y aprobado para su realización.

Análisis estadístico: Se resumieron las variables continuas en mediana y rango intercuartilo (RIC) y las categorías, en frecuencia y porcentaje. Se compararon las características clínicas y de laboratorio al momento de la admisión de los pacientes según tuvieran bacteriemia o no. Se realizó un análisis bivariado. Se utilizó el test de Ranksum para las variables continuas con distribución no normal y el test de chi cuadrado para las categóricas para comparar ambos grupos. Se consideró estadísticamente significativo cuando $p$ fuera menor de 0,05. Se utilizó Stata 10. 


\section{RESULTADOS}

Fueron incluidos 62 pacientes con IOA que cumplieron con los criterios de inclusión. Eran varones 34 pacientes (55\%). La mediana de edad fue de 59,5 meses (RIC 24-84).

El tiempo de evolución previa tuvo una mediana de 3 días (RIC 1-6). Habían tenido fiebre antes de internarse 44 pacientes $(70 \%)$. El motivo de consulta fue, en 48 pacientes $(77 \%)$, dolor e impotencia funcional. Referían haber recibido algún antibiótico en la semana previa a la internación 4 pacientes $(6 \%)$.

Los pacientes se presentaron con artritis (54 pacientes, $87 \%$ ), osteoartritis (6 pacientes, $10 \%$ ) y osteomielitis (n: $2 ; 3 \%$ ) (Tabla 1).
La localización de las infecciones fue en la rodilla en 29 pacientes (47\%), en la cadera en 23 pacientes $(37 \%)$, en el tobillo en 7 pacientes $(11 \%)$, en el hombro en $3(4 \%)$, en el fémur en $3(4 \%)$, en la tibia en $1(2 \%)$ y en el peroné en $1(2 \%)$. Solo 5 pacientes $(8 \%)$ tuvieron compromiso poliarticular.

$\mathrm{Al}$ ingresar, presentaban en el hemograma una mediana de 13000 glóbulos blancos (RIC 10 400-16600). La mediana de proteína C reactiva (PCR) inicial fue de 47 U/L (RIC 19-100) y eritrosedimentación de $71 \mathrm{~mm} / \mathrm{h}$ (RIC 44-93).

Se realizaron hemocultivos y cultivo de líquido articular o biopsia ósea en todos los pacientes incluidos. Se identificó algún agente etiológico en 25 pacientes $(40 \%)$. Predominó Staphylococcus aureus (n: 20, 32\%) (Tabla 2).

TABLA 1. Características clínicas y de laboratorio registradas en el momento de la admisión en niños con infección osteoarticular con y sin bacteriemia

\begin{tabular}{|c|c|c|c|c|}
\hline \multicolumn{2}{|l|}{ Característica } & $\begin{array}{c}\text { Niños con bacteriemia (n: 15) } \\
\mathrm{N} \%\end{array}$ & $\begin{array}{c}\text { Niños sin bacteriemia (n: 47) } \\
\text { N \% }\end{array}$ & $P$ \\
\hline \multicolumn{2}{|l|}{ Sexo masculino } & $8(53)$ & $26(55)$ & 0,9 \\
\hline \multicolumn{2}{|c|}{ Edad en meses (mediana, RIC) } & $26(12-90)$ & $60(24-85)$ & $0,02^{*}$ \\
\hline \multicolumn{2}{|c|}{ Días de evolución hasta la consulta (mediana, RIC) } & $3(1-5)$ & $3(1-7)$ & 0,6 \\
\hline \multicolumn{2}{|c|}{ Uso de antibiótico previo } & - & $4(9)$ & - \\
\hline \multicolumn{2}{|c|}{ Recuento de glóbulos blancos (/ $\left.\mathrm{mm}^{3}\right)$ (mediana, RIC) } & $13100(9400-17700)$ & $13000(11000-16000)$ & 0,8 \\
\hline \multicolumn{2}{|c|}{ Hemoglobina $(g / d l)$} & $10,8(9,9-12)$ & $12,5(10-13)$ & $0,04^{*}$ \\
\hline \multicolumn{2}{|c|}{ Recuento de plaquetas $\left(/ \mathrm{mm}^{3}\right)$ (mediana, RIC) } & $281000(154000-346000)$ & $340000(288000-341000)$ & 0,07 \\
\hline \multicolumn{2}{|c|}{ Eritrosedimentación $\left(\mathrm{mm}^{3}\right)$ (mediana, RIC) } & $65(45-83)$ & $73(43-95)$ & 0,7 \\
\hline \multicolumn{2}{|c|}{ Proteína C reactiva (U/L) (mediana, $\mathrm{RIC})$} & $101(57-203)$ & $33(17-56)$ & $0,01^{*}$ \\
\hline \multicolumn{2}{|c|}{ Fiebre } & $100(15)$ & $57(28)$ & $<0,01$ \\
\hline \multirow[t]{3}{*}{ Diagnóstico final: } & Artritis & $12(80)$ & $42(89)$ & 0,6 \\
\hline & Osteoartritis & $3(20)$ & $3(6)$ & $0,03^{*}$ \\
\hline & Osteomielitis & $0(0)$ & $2(4)$ & - \\
\hline
\end{tabular}

* Estadísticamente significativo.

RIC: rango intercuartilo.

TABLA 2. Identificación microbiológica según el sitio de documentación. N: 25

\begin{tabular}{|c|c|c|}
\hline Sitio de identificación & Germen & $\mathbf{N}(\%)$ \\
\hline Hemocultivos N: 5 & $\begin{array}{l}\text { Staphylococcus aureus meticilino resistente } \\
\text { Staphylococcus aureus meticilino sensible }\end{array}$ & $\begin{array}{l}4(16) \\
1(4)\end{array}$ \\
\hline Hemocultivos y líquido articular o biopsia ósea N: 10 & $\begin{array}{l}\text { Staphylococcus aureus meticilino resistente } \\
\text { Staphylococcus aureus meticilino sensible } \\
\text { Neisseria meningitidis } \\
\text { Streptococcus pyogenes }\end{array}$ & $\begin{array}{l}5(20) \\
3(12) \\
1(4) \\
1(4)\end{array}$ \\
\hline Líquido articular N: 9 & $\begin{array}{l}\text { Staphylococcus aureus meticilino resistente } \\
\text { Staphylococcus aureus meticilino sensible } \\
\text { Streptococcus pyogenes }+ \text { S. aureus meticilino resistente } \\
\text { Haemophilus influenzae } \\
\text { Kingella kingae } \\
\text { Moraxella catarrhalis }\end{array}$ & $\begin{array}{l}3(12) \\
2(8) \\
1(4) \\
1(4) \\
1(4) \\
1(4)\end{array}$ \\
\hline Biopsia ósea N: 1 & Staphylococcus aureus meticilino resistente & $1(4)$ \\
\hline Líquido articular y biopsia ósea N: 1 & Pseudomonas aeruginosa & $1(4)$ \\
\hline
\end{tabular}


Quince pacientes tuvieron bacteriemia (24\%). Las bacterias identificadas en hemocultivos fueron SAMR (n: 9; 14\%), Staphylococcus aureus meticilino sensible (n: $4 ; 6 \%$ ), Neisseria meningitidis (n: $1 ; 2 \%)$ y Streptococcus pyogenes (n: $1 ; 2 \%$ ).

El tratamiento empírico fue clindamicina (30 mg $/ \mathrm{kg} /$ día) en 56 pacientes (90\%), vancomicina (40 mg/ kg/ día) en $3(5 \%)$ y ceftriaxona (50 mg/ $\mathrm{kg} /$ día) en $2(3 \%)$. En un paciente, no se indicó tratamiento empírico, ya que se interpretó como una sinovitis transitoria. La mediana de duración del tratamiento endovenoso fue 7 días (RIC 5-11). La mediana de internación fue 7 días (RIC 4-12). El tratamiento definitivo fue clindamicina en 38 pacientes $(61 \%)$, trimetoprima/ sulfametoxazol en $11(18 \%)$, cefalexina en $6(10 \%)$, amoxicilina en $3(5 \%)$ y ciprofloxacina en $1(2 \%)$. En 2 pacientes $(4 \%)$, se indicó tratamiento oral combinado: amoxicilina y trimetoprima/sulfametoxazol. La mediana de duración total del tratamiento (parenteral oral) fue 30 días (RIC 20-42). Solo 1 paciente $(2 \%)$ presentó una complicación en la evaluación a 3 meses (fractura patológica).

Se compararon las características de los pacientes con bacteriemia con los que no la presentaron. En el análisis bivariado (Tabla 1), se identificó menor edad en los niños con hemocultivos positivos (26 meses vs. 60, $\mathrm{p}<0,05$ ), mayor valor de PCR inicial (101 vs. $33 \mathrm{U} / \mathrm{L}, \mathrm{p}<0,05$ ), hemoglobina $(10,8 \mathrm{~g} / \mathrm{dl}$ vs. $12,5 \mathrm{~g} / \mathrm{dl}, \mathrm{p} 0,04)$ y mayor frecuencia de fiebre $(100 \%$ vs. $57 \%, p<0,05)$. No se encontraron diferencias estadísticamente significativas en el análisis bivariado entre ambos grupos en el sexo, días de evolución hasta la consulta, valores de eritrosedimentación, recuento de glóbulos blancos, hemoglobina y plaquetas al momento de la internación.

Ningún paciente falleció ni requirió admisión en la Unidad de Cuidados Intensivos.

\section{DISCUSIÓN}

Las IOA son infecciones graves que requieren manejo adecuado precoz para mejorar el pronóstico. A partir del uso universal de vacunas conjugadas en menores de 2 años y del aumento de la incidencia de infecciones por SAMR adquirido en la comunidad, en pediatría, se reporta mundialmente un cambio en la epidemiología de las IOA. ${ }^{7}$

La mediana de edad de presentación reportada en la literatura de las IOA en niños varía de 36 meses $^{8}$ a 7 años, ${ }^{5}$ y son mayores los pacientes con infecciones por SAMR, como en el estudio presentado.
La forma de presentación más frecuente es como monoartritis aguda, aunque se reporta compromiso de más de una articulación hasta en un $22 \%$ de los casos. ${ }^{9}$ En la cohorte presentada, solo hubo compromiso poliarticular en el $8 \%$ de los pacientes.

Las grandes articulaciones se encuentran comprometidas con mayor frecuencia que las pequeñas. Las localizaciones más frecuentes en niños son, coincidentes con nuestra serie, cadera y rodilla. ${ }^{10}$

En una revisión sistemática realizada por Margaretten et al., ${ }^{11}$ se evaluó la utilidad diagnóstica de las características clínicas para el diagnóstico de las IOA bacterianas. Se reportó que la fiebre, los hallazgos del hemograma, los valores de eritrosedimentación y la PCR no eran suficientes para excluir el diagnóstico de infección bacteriana. En nuestro estudio, la PCR se asoció estadísticamente con los hemocultivos positivos.

La clave para el diagnóstico ante la sospecha clínica de IOA son los estudios microbiológicos y de imágenes. En el 50-80\% de los casos, se documenta el germen implicado. ${ }^{12}$ La tinción de Gram del líquido sinovial y la microscopía dan un resultado positivo en solo un $50 \%$ de los casos de artritis séptica. ${ }^{13}$ Los hemocultivos son positivos en el $60 \%$ de los casos de cuadros agudos. En nuestra cohorte, se identificó algún agente etiológico en el $40 \%$ de los casos.

Los patógenos predominantes en las IOA de los niños son Staphylococcus aureus y Streptococcus pyogenes. ${ }^{14}$ Antes de la introducción de la vacuna conjugada contra Haemophilus influenzae b, las IOA representaban una de las formas de presentación de las infecciones invasivas por este bacilo Gramnegativo. Sin embargo, a partir del uso universal de la vacuna conjugada, la incidencia de este tipo de infecciones disminuyó rotundamente. ${ }^{15}$

No obstante, el gran cambio en la epidemiología de las IOA ha sido la emergencia de SAMR en la comunidad. En las últimas décadas, se describe un amplio predominio de SAMR en las IOA en Argentina, coincidente con los hallazgos de este estudio. ${ }^{16}$

El tratamiento quirúrgico y antibiótico precoz mejora la evolución de las IOA agudas. Para la elección del antibiótico empírico, debe considerarse que, en nuestro medio, predomina ampliamente SAMR en este tipo de infecciones. ${ }^{17}$

Diferentes estudios evaluaron las características diferenciales de los niños con IOA con y sin bacteriemia. ${ }^{18,19}$ En un estudio realizado en Finlandia en pacientes con IOA con aislamiento de 
Staphylococcus aureus meticilino sensible, la PCR fue el único marcador significativamente mayor en los niños con hemocultivos positivos. Al igual que en el trabajo realizado, no se identificaron diferencias en los valores de eritrosedimentación ni recuento de glóbulos blancos.

Otro estudio ${ }^{20}$ realizado en una población con alta prevalencia de resistencia a la meticilina reportó mayor duración de la fiebre, bacteriemia, prolongación de la internación y más procedimientos quirúrgicos en los niños con IOA con bacteriemia por SAMR. Categorizar a los pacientes con mayor riesgo de bacteriemia permite identificar precozmente a los niños que requerirán más días de internación y plantear drenajes quirúrgicos precoces y adecuados.

Se reporta, en la literatura, hasta un $20 \%$ de niños con secuelas al año. ${ }^{21}$ Los pacientes con mayor tiempo de evolución antes de la consulta, retraso en el tratamiento, reactantes de fase aguda aumentados, ${ }^{22}$ menor edad e identificación de SAMR han sido descritos como factores de mal pronóstico. ${ }^{23}$ Dado que este estudio no fue diseñado para evaluar la morbilidad a largo plazo de las IOA en niños, el seguimiento no fue lo suficientemente prolongado como para poder evaluar secuelas y sacar conclusiones definitivas.

Los estudios sobre IOA en niños realizados en nuestro país en los últimos años son escasos. Una de las principales fortalezas de este trabajo es el número considerable de pacientes incluidos con sospecha de IOA en un corto período y en un solo centro. En todos los casos, los pacientes fueron evaluados en forma sistemática con hemocultivos y líquido sinovial. Se excluyeron los pacientes con historia de internación reciente para mejorar la validez externa de los hallazgos.

Sin embargo, debe considerarse que el ámbito de investigación de este estudio es un hospital pediátrico de alta complejidad donde se derivan pacientes de todo el país. Son pacientes que suelen requerir procedimientos diagnósticos y terapéuticas específicas que no están disponibles en otros centros asistenciales. Estas características deben considerarse antes de generalizar los hallazgos del estudio.

Se requieren estudios que incluyan mayor número de pacientes y permitan explorar las variables clínicas y de laboratorio en un análisis multivariado.

\section{CONCLUSIONES}

En esta cohorte de niños con IOA, se identificó SAMR como el primer agente etiológico.
Los niños con bacteriemia tuvieron menor edad, mayor valor de PCR y más frecuentemente fiebre que los que se presentaron con hemocultivos negativos.

\section{REFERENCIAS}

1. Paganini HR. Artritis séptica. En: Infectología pediátrica. Buenos Aires: Científica interamericana;2007.Págs.565-75.

2. Rosanova MT.Infecciones osteoarticulares: Artritis sépticas, Osteomielitis. En: Bruno M, Marcó del Pont J, Ellis A, et al. Libro Azul Infectología Pediátrica. 3. ${ }^{\text {ra }}$ ed. Buenos Aires: FUNDASAP; 2007.Págs.178-86.

3. Rosanova MT. Infecciones osteoarticulares. Artritis séptica. Med Infant. 2008;15(4):342-6.

4. Hernández T, Zarzoso S, Navarro M, et al. Osteomielitis y artritis séptica. Protocolos diagnóstico-terapéuticos de la AEP: Infectología pediátrica. Madrid: Hospital Materno-Infantil Gregorio Marañón; 2010. [Acceso: 30 de abril de 2017]. Disponible en http: / / www.aeped.es / sites / default/files / documentos / osteomielitis.pdf.

5. McNeil JC, Kaplan SL, Vallejo JC. The Influence of the Route of Antibiotic Administration, Methicillin Susceptibility, Vancomycin Duration and Serum Trough Concentration on Outcomes of Pediatric Staphylococcus aureus Bacteremic Osteoarticular Infection. Pediatr Infect Dis J 2017;36(6):572-7.

6. Clinical and Laboratory Standards Institute. Performance Standards for AntimicrobialSusceptibility Testing; TwentyFifth InformationalSupplement. CLSI documentM100-S25. Wayne, PA: CLSI; 2015.

7. Arnold SR, Elias D, Buckingham SC, et al. Changing patterns of acute hematogenous osteomyelitis and septic arthritis: emergence of communityassociated methicillin-resistant Staphylococcus aureus. J Pediatr Orthop 2006;26(6):703-8.

8. Bologna R. Tratamiento oral de infecciones severas. Med Infant. 1996;3(3):170-4.

9. Dubost JJ, Fis I, Denis P, et al. Polyarticular septic arthritis. Medicine (Baltimore) 1993;72(5):296-310.

10. Pääkkönen M, Peltola H. Treatment of acute septic arthritis. Pediatr Infect Dis J 2013;32(6):684-5.

11. Li SF, Cassidy C, Chang C, et al. Diagnostic utility of laboratory tests in septic arthritis. Emerg Med J 2007; 24(2):75-7.

12. Mathews CJ, Coakley G. Septic artritis: current diagnostic and therapeutic algorithm. Current Opin Reumatol 2008;20(4):457-62.

13. Mathews CJ, Kingsley G, Field M, et al. Management of septic arthritis: a systematic review. Ann Rheum Dis 2007;66(4):440-5.

14. Gupta MN, Sturrock RD, Field M. A prospective 2-year study of 75 patients with adult-onset septic arthritis. Rheumatology (Oxford) 2001;40(1):24-30.

15. Howard AW, Viskontas D, Sabbagh C. Reduction in osteomyelitis and septic arthritis related to Haemophilus influenzae type B vaccination. J Pediatr Orthop 1999; 19(6):705-9.

16. Rosanova MT, Berberian G, Bologna R, et al. Estudio descriptivo de infecciones osteo-articulares en niños en tiempos de Staphylococcus aureus resistente a meticilina de la comunidad (SARM-Co). Rev Chil Infectol 2015;32(3):321-5.

17. Rosanova MT, Berberian G, Ruvinsky S, et al. Infecciones por Staphylococcus Aureus Meticilino resistente de la comunidad. Tratamiento basado en la evidencia. Med Infant 2011;18(1):76-8.

18. Pääkkönen M, Kallio MJ, Kallio PE, et al. C-reactive protein versus erythrocyte sedimentation rate, white blood cell count and alkaline phosphatase in diagnosing 
bacteraemia in bone and joint infections. J Paediatr Child Health 2013;49(3):E189-92.

19. Peltola H, Räsänen JA. Quantative C-reactive protein in relation to erythrocyte sedimentation rate, fever, and duration of antimicrobial therapy in bacteraemic diseases of childhood. J Infect 1982;5:257-67.

20. McNeilJ,Hulten K, MasonE, etal.Bacteremic Staphylococcus aureus Osteoarticular Infections: Observations on Treatment and Outcomes in a Population With a High Prevalence of Methicillin-Resistant Staphylococcus aureus (MRSA). Open Forum Infect Dis 2016;3(Suppl 1): 673.
21. Howard-Jones AR, Isaacs D, Gibbons PJ. Twelve-month outcome following septic arthritis in children. J Pediatr Orthop B 2013;22(5):486-90.

22. Yuan HC, Wu KG, Chen CJ, et al. Characteristics and outcome of septic arthritis in children. J Microbiol Immunol Infect 2006;39(4):342-7.

23. Vander Have KL, Karmazyn B, Verma M, etal. Communityassociated methicillin-resistant Staphylococcus aureus in acute musculoskeletal infection in children: a game changer. J Pediatr Orthop 2009;29(8):927-31. 